\title{
Erratum: Hadron fragmentation inside jets in hadronic collisions [Phys. Rev. D 92, 054015 (2015)]
}

\author{
Tom Kaufmann, Asmita Mukherjee, and Werner Vogelsang
}

(Received 20 March 2020; accepted 23 March 2020; published 7 April 2020)

DOI: $10.1103 /$ PhysRevD.101.079901

The partonic jet functions describing the inclusive production of jets or hadronic fragmentation inside jets were shown to obey momentum sum rules [1,2]. Reference [3] pointed out that in the case of cone-based jet algorithms previously available next-to-leading-order (NLO) jet functions did, however, not obey these momentum sum rules. This was traced back to a mistake in the previous computations of the jet functions, and corrected jet functions were given.

These recent developments also entail corrections for some of our results presented in this paper. Our inclusive jet functions given in Eq. (12) are corrected as (for details, see Ref. [4])

$$
\mathcal{J}_{i}\left(z, \lambda \equiv \frac{\mathcal{R} p_{T}^{\text {jet }}}{\mu_{F}^{\prime}}, \mu_{R}\right)=\mathcal{J}_{i}^{\text {Eq. }(12)}\left(z, \lambda, \mu_{R}\right)+\frac{\alpha_{s}\left(\mu_{R}\right)}{2 \pi} \mathcal{I}_{i}^{\text {algo }}(z)
$$

where for the cone algorithm,

$$
\begin{gathered}
\mathcal{I}_{q}^{\text {cone }}(z)=2\left(P_{q q}(z)+P_{g q}(z)\right)\left[\ln (z) \Theta\left(z-\frac{1}{2}\right)+\ln (1-z) \Theta\left(\frac{1}{2}-z\right)\right], \\
\mathcal{I}_{g}^{\text {cone }}(z)=2\left(P_{g g}(z)+2 N_{f} P_{q g}(z)\right)\left[\ln (z) \Theta\left(z-\frac{1}{2}\right)+\ln (1-z) \Theta\left(\frac{1}{2}-z\right)\right],
\end{gathered}
$$

corresponding to the results given in Ref. [3], while for the " $J_{E_{T}}$ " algorithm, $\mathcal{I}_{i}^{J_{E_{T}}}(z)=\frac{1}{2} \mathcal{I}_{i}^{\text {cone }}(z)$, and for the (anti-) $k_{t}$ algorithms, $\mathcal{I}_{q}^{k_{t}}=\mathcal{I}_{g}^{k_{t}}=0$. For the "jet size" parameter $\mathcal{R}$, we have $\mathcal{R}=R$ for the cone and (anti-) $k_{t}$ algorithms, and $\mathcal{R}=1 / \sqrt{\beta}$ for the $J_{E_{T}}$ algorithm (the latter value correcting the one given in our paper).

We note that with these changes there is a simple relation among the jet functions for the various algorithms. For a given value of $\mathcal{R}$, we have

$$
\mathcal{J}_{i}^{J_{E_{T}}}\left(z, \lambda, \mu_{R}\right)=\frac{1}{2}\left[\mathcal{J}_{i}^{\text {cone }}\left(z, \lambda, \mu_{R}\right)+\mathcal{J}_{i}^{k_{t}}\left(z, \lambda, \mu_{R}\right)\right]
$$

In the same way as above, our jet functions for hadron fragmentation inside jets also need to be revised. The functions $j_{c \rightarrow c^{\prime}}\left(z, \lambda, \mu_{R}\right)$ in Eqs. (23) are corrected as

$$
j_{c \rightarrow c^{\prime}}\left(z, \lambda, \mu_{R}\right)=j_{c \rightarrow c^{\prime}}^{\text {Eq. }(23)}\left(z, \lambda, \mu_{R}\right)+\frac{\alpha_{s}\left(\mu_{R}\right)}{2 \pi} \tilde{\mathcal{I}}_{c^{\prime} c}^{\text {algo }}(z),
$$

where for the cone algorithm

$$
\tilde{\mathcal{I}}_{c^{\prime} c}^{\text {cone }}(z)=2 P_{c^{\prime} c}(z)\left[\ln (z) \Theta\left(z-\frac{1}{2}\right)+\ln (1-z) \Theta\left(\frac{1}{2}-z\right)\right]
$$

while for the $J_{E_{T}}$ algorithm $\tilde{\mathcal{I}}_{c^{\prime} c}^{J_{E_{T}}}(z)=\frac{1}{2} \tilde{\mathcal{I}}_{c^{\prime} c}^{\text {cone }}(z)$ and for the (anti-) $k_{t}$ algorithms $\tilde{\mathcal{I}}_{c^{\prime} c}^{k_{t}}(z)=0$. These results easily translate to the functions $\mathcal{K}_{c \rightarrow c^{\prime}}\left(z, z_{p}, \lambda, \kappa, \mu_{R}\right)$ in Eqs. (21) and (B.1)-(B.3).

None of the numerical results presented in our paper is affected by these changes since for the phenomenological part we only considered the (anti-) $k_{t}$ algorithm, for which no corrections occur. 
We are grateful to Zhongbo Kang and Felix Ringer for very useful discussions.

[1] L. Dai, C. Kim, and A. K. Leibovich, Phys. Rev. D 94, 114023 (2016).

[2] M. Procura and W. J. Waalewijn, Phys. Rev. D 85, 114041 (2012).

[3] Z. B. Kang, F. Ringer, and W. J. Waalewijn, J. High Energy Phys. 07 (2017) 064.

[4] T. Kaufmann, Ph.D. thesis, University of Tübingen, 2017. 